\title{
New approaches to solve the old Blazhko puzzle in RR Lyrae stars
}

\author{
K. Kolenberg \\ ${ }^{1}$ Institute for Astronomy, University of Vienna, Türkenschanzstrasse 17, A - 1180 Wien, \\ Austria \\ email: kolenberg@astro.univie.ac.at
}

\begin{abstract}
Almost a century after its discovery, the phenomenon of amplitude and/or phase modulation, observed in a large percentage of the RR Lyrae stars, still lacks a widely accepted theoretical understanding. Recent attempts to theoretically explain the effect focus on two alternatives, the magnetic models and the resonances models, both involving the presence of nonradial pulsation components.

We present the 'Blazhko project', a large international collaboration focused on understanding the Blazhko effect. The aim of the 'Blazhko project' is to combine spectroscopic and photometric data from a sample of well-selected Blazhko and nonBlazhko stars, to reveal decisive information on the physical mechanism responsible for the modulation.
\end{abstract}

Keywords. Stars: oscillations; stars: variables: RR Lyrae, stars: horizontal-branch

\section{RR Lyrae stars and the Blazhko effect}

The study of RR Lyrae stars is a particularly active field of research, with numerous present and future applications. These pulsating variables have mean periods of less than a day, and show brightness variations of the order of a magnitude. Until not so long ago, RR Lyrae stars were considered to be prototypes of purely radial pulsators.

The most intriguing subclass of RR Lyrae stars consists of stars showing the Blazhko effect, the phenomenon of amplitude and/or phase modulation, which was named after one of its discoverers (Blazhko 1907). The lightcurve of a Blazhko star periodically changes its shape over a timescale of typically tens to hundreds of days (Figure 1). There seems to be a lack of correlation between the pulsation period and the Blazhko period.

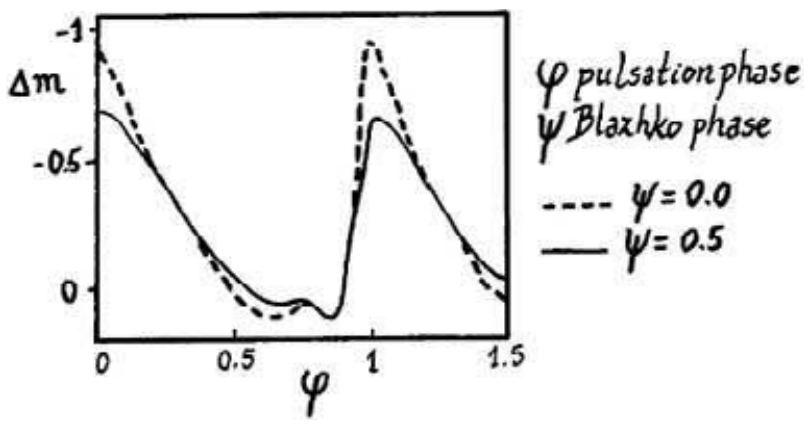

Figure 1. Illustration of the light curve changes of a Blazhko star.

The estimated incidence rate of Blazhko variables among the galactic RRab stars (fundamental mode pulsators) is about 20-30.\% (Szeidl 1988, Moskalik \& Poretti 2002). For the RRc Blazhko stars (first overtone pulsators) this rate is less than $5 \%$. In the 
Large Magellanic Cloud the incidence rate for RRab stars is only half as large, which is probably a metallicity effect (Alcock et al. 2003).

\section{Past and present studies of the Blazhko effect}

The Blazhko effect has been the frequent subject of photographic and photometric studies (e.g., Szeidl \& Kollath 2000). Rigorous frequency analyses of photometric data for Northern field Blazhko stars were carried out by, e.g., Borkowski (1980), Kovacs (1995), and Smith et al. (2003), just to mention a few. For RR Lyr, the brightest Blazhko star, important spectroscopic studies were carried out by Struve \& Blaauw (1948) and Preston et al. (1965).
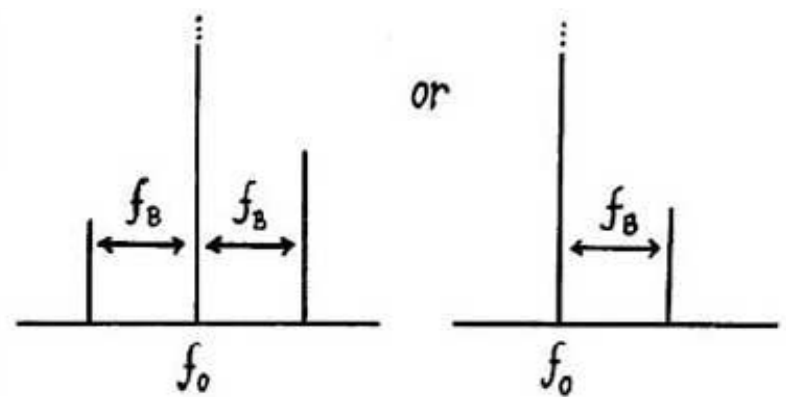

Figure 2. Triplet or doublet in the frequency spectrum of a Blazhko star.

In the past decade systematic studies of accurate CCD data of globular clusters and the Magellanic Clouds (e.g., MACHO, OGLE) have cast a new light upon the study of RR Lyrae variability (Moskalik \& Poretti 2003, Alcock et al. 2000, 2003), and have yielded important statistics on the phenomenology of the Blazhko effect. The frequency spectra of light and radial velocity curves of RR Lyrae Blazhko stars exhibit either a doublet structure or an equally-spaced triplet structure around the main pulsation frequency and its harmonics, with a small frequency separation corresponding to the Blazhko frequency (Figure 2). The observed period ratios are 0.95-1.05, which excludes the possibility of another radial mode being excited. A large majority of the Blazhko stars have a larger modulation peak at the higher (rather than the lower) frequency side of the main pulsation component. The Blazhko effect is not found among the long period RRab stars (Smith 1981). Blazhko stars at their greatest light amplitude fall approximately on the curve of amplitude versus period as defined by stars with regular light curves. This indicates that the Blazhko effect tends to reduce the maximum light level.

Period changes are a common feature in RR Lyrae stars, and also occur in Blazhko stars (Smith 1995, Szeidl \& Kollath 2000, LaCluyzé et al. 2002). The observed period variability is too fast to be of evolutionary nature. In some stars the Blazhko effect ceased. Some well-studied field Blazhko stars are reported to display, besides their Blazhko cycles, also very long periods of the order of years. RR Lyrae, for example, shows a cycle of about 4 years, at the end of which the strength of the modulation suddenly decreases, and a phase shift of about 10 days occurs in the Blazhko cycle. This phenomenon is still unexplained, though it has reminded some investigators of the solar magnetic cycle.

\section{Explanations for the Blazhko effect in RR Lyrae stars}

The most plausible hypotheses to explain the phenomenon focus on two types of models, both involving nonradial pulsation components (Figure 3). 

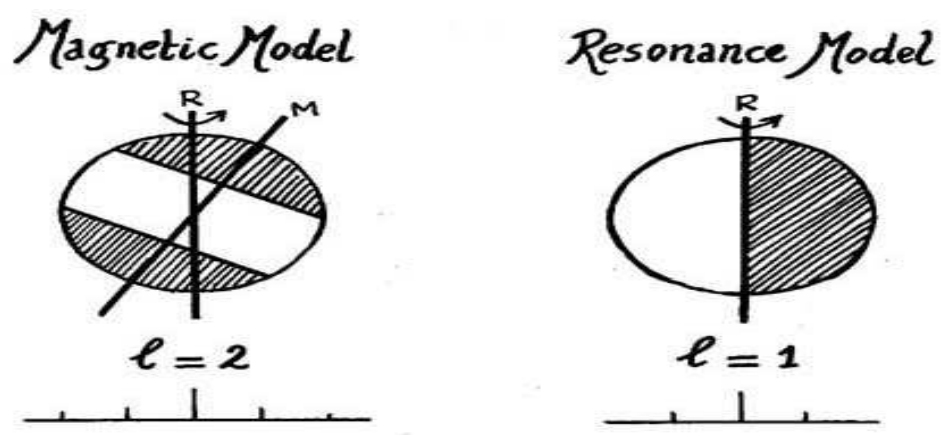

Figure 3. The two prevailing models for the Blazhko effect.

\subsection{The resonance models}

The resonance models are based on a (nonlinear) resonance between the radial fundamental mode and a nonradial mode. In these models the dipole $(\ell=1)$ modes have the highest probability to be nonlinearly excited (Cox 1993, Van Hoolst et al. 1998). Nowakowski \& Dziembowski 2001) predict significant amplitude and phase modulation in the case of excitation of a rotationally split $m= \pm 1$ pair. The modulation period is determined by the rotation rate (currently unknown) and the Brunt-Väisälä frequency in the deepest part of the radiative interior. Peterson (1996) measured the line-widths via cross-correlation for $27 \mathrm{RR}$ Lyrae stars and obtained an upper limit for $v \sin i$ of $10 \mathrm{~km} \mathrm{~s}^{-1}$.

\subsection{The magnetic models}

The magnetic models, like the simple oblique pulsator model for roAp stars (Kurtz 1982), suppose that Blazhko stars have a magnetic field inclined to the stellar rotation axis (Cousens 1983, Shibahashi \& Takata 1995). The main radial mode is deformed by the magnetic field to have an additional quadrupole component $(\ell=2)$, whose symmetry axis coincides with the magnetic axis. Due to the star's rotation our view of the pulsation components changes, causing the observed amplitude modulation. Shibahashi \& Takata (1995) predicted a quintuplet structure in the frequency spectrum, but also showed that the quintuplet looks almost like a triplet for certain geometrical configurations. Depending on which of the side components we then observe, the Blazhko period is supposed to be equal to the rotation period or half of the rotation period. According to the latest model, a magnetic field of about $1 \mathrm{kG}$ is needed in this model for the amplitude modulation to be observable. Babcock (1958) and Romanov et al. (1994) reported a variable magnetic field in RR Lyr with a strength up to $1.5 \mathrm{kG}$, whereas Preston (1967) and Chadid et al. (2001, 2004) contradict these measurements.

Despite the progress made in the last decade both in observations and modelling, a basic physical understanding of the Blazhko phenomenon is still lacking. Unsolved questions involve the modulation components of unequal amplitudes, the deviations from strict amplitude/phase modulation, the different incidence rates for fundamental mode and first overtone pulsators, the role of metallicity, the role of convective turbulence, etc. As all current models for explaining the Blazhko effect are based on the presence of nonradial components, their detection and identification is of utmost importance for understanding the mechanism behind the amplitude modulation. 


\section{New developments in line profile analysis}

Up to now most observational studies of Blazhko stars were based on photometric data. High-resolution line profiles offer much better diagnostics to find and identify nonradial oscillation components in pulsating stars. A few years ago the first line profile study aiming at an identification of the nonradial mode(s) was carried out by Kolenberg et al. (2003). It was based on a set of 669 high-resolution $(R=40000)$ spectra of $\mathrm{RR}$ Lyr, obtained with the spectrograph ELODIE attached to the $1.93-\mathrm{m}$ telescope at the Observatoire de Haute-Provence in France (Chadid et al. 1999). A detailed study of the variations of the Fe II $\lambda 4923.921$ led to a clear detection of nonradial pulsation components in the star. By means of an adapted version of the moment method (Balona 1986, Aerts 1996, Kolenberg 2002), the detected nonradial modes were identified as nonaxisymmetric (with respect to the rotation axis) modes of degree $\ell \leqslant 3$. The incomplete coverage of the data over the Blazhko cycle hampered a more precise identification. As this kind of analysis is a first essential step towards a decisive confrontation between the theoretical models and the observations, more spectroscopic data, additional data, better spread over the Blazhko cycle, and similar data sets of additional (Blazhko) RR Lyrae stars are highly desired.

\section{The Blazhko project}

The Blazhko project is a large international collaboration, set up to join efforts in obtaining a better understanding of the Blazhko phenomenon in RR Lyrae stars. The project was founded in Vienna and started its activities in the autumn of 2003.

\subsection{Methodology}

The starting point for improving the modelling is an extensive database for a limited sample of field RR Lyr Blazhko and nonBlazhko stars. We selected a few RRab Blazhko stars, in the Northern and in the Southern hemisphere, and one RRc Blazhko target. Important also is the inclusion of a few well-selected, nonmodulated RR Lyrae stars in the target list, of which similar data are being gained, to be compared with the Blazhko stars.

The required data set consists of high-resolution $(R \geqslant 40000)$, high- $S / N(\geqslant 100)$ spectroscopic data evenly spread over the Blazhko cycle for the target stars. A few very detailed snapshots $(S / N \geqslant 200)$ are being (and will be) obtained with telescopes of the 8-m class, and will help to distinguish between different nonradial modes. We carried out a simulation study to find out how much high-resolution data are minimally needed to be able to disentangle the modes, and what would be their optimal time spread. Additional radial velocities over a longer time base can be obtained with smaller telescopes and provide essential information to interpret the line profile variations. Finally, photometric data gathered over a time base of at least a year, are needed to ensure the required frequency resolution. For the interpretation of the data we will use the available spectroscopic identification methods, as well as combined techniques presently being developed in Vienna (see Zima et al. 2004).

\subsection{Present status}

At the time of writing the project has about 25 collaborators, observational and theoretical astronomers, in both hemispheres.

In 2004, an extensive spectroscopic and photometric campaign was dedicated to RR Lyrae, gathering observations from 10 different observatories. For the Southern hemisphere, a sample of relatively unstudied Blazhko stars is being sampled photometrically, 
with the aim of determing Blazhko periods and selecting the best targets for a spectroscopic study.

From the theoretical side, line profile variations are being studied for stars pulsating in a radial mode, modulated by nonradial components with close frequencies. These experiments also serve to determine the optimal time spread for the observations, to make efficient use of telescope time. Theoretical studies may also reveal how light curves are influenced at maximum/minimum light by the interaction of different modes. Finally, spectroscopic mode identification methods and their applicability are being tested in a Vienna-Leuven collaboration (Zima et al. 2004)

The status of the Blazhko project can be checked on a new website dedicated to the collaboration: http://www.astro.univie.ac.at/ ${ }^{\mathrm{d} s n}$.

\section{Acknowledgements}

I would like to acknowledge the International Astronomical Union and the SOC for awarding me a IAU grant to participate in this Symposium. I also thank for useful suggestions and comments on the topic given during the conference, and for the positive reactions, which in the meantime have given rise to fruitful collaboration. This project is supported by the Austrian Research Foundation (Wissenschafstfonds, FWF grant P17097-N02).

\section{References}

Aerts, C. 1996, A\&A, 314, 115

Alcock, C., Allsman, R., Alves, D. R., Axelrod, T., Becker, A., Bennett, D., Clement, C., Cook, K. H., Drake, A., Freeman, K., \& the MACHO collaboration 2000, ApJ 542, 257

Alcock, C., Alves, D. R., Becker, A., Bennett, D., Cook, K. H., Drake, A., Freeman, K., Geha, M., Griest, K., Kovács, G., \& the MACHO collaboration 2003, ApJ 542, 257

Babcock, H.W. 1958, ApJS, 3, 141

Balona, L.A. 1986, MNRAS, 219, 111

Blazhko, H.W. 1907, Astron. Nachr. 175, 325

Borkowski, K.J. 1980 Acta Astron. 30, 393

Chadid, M., Kolenberg, K., Aerts, C., Gillet, D. 1999, A\&\&A 352, 201

Chadid, M., Wade, G.A., Shorlin, S.L.S., Landstreet, J.D. 1999, A $\& A$ 413, 1087

Cousens, A. 1983, MNRAS, 203, 1171

Cox, A.N. 1993, Proc. IAU Coll. 139, 409

Kolenberg, K. 2002, PhD Thesis, Leuven University, Belgium, http://www.ster.kuleuven . ac.be/pub/kolenberg_phd/

Kolenberg, K., Aerts, C., Fokin, A., Dziembowski, W., Chadid, M., Gillet, D. 2003, ASP Conf. Ser. 259,396

Kovacs, G. 1995, A\&BA, 295, 693

Kovacs, G. 2002, ASP Conf. Ser. 259, 396

Kurtz, D.W. 1982, MNRAS 200, 807

LaCluyzé, A., Smith. H.A., Gil, E.-M., Hedden, A., Kinemuchi, K. Rosas, A. M., Pritzl, B. J., Sharpee, B., Robinson, K. W., et al. 2002, ASP Conf. Ser. 259, 416

Moskalik, P. \& Poretti, E. 2003, A\&广A 398, 213

Nowakowski, R.M. \& Dziembowski, W.A. 2001, Acta Astron., 51, 5

Peterson, R.C., Carney, B.W., Latham, D.W. 1996, ApJ, 465, 47

Preston, G.W., Smak, J., Paczyński, B. 1965, ApJS, 12, 99

Shibahashi, H., Takata, M. 1995, ASP Conf. Ser. 83, 42

Smith. H.A. 1981, PASP, 93, 721

Smith. H.A. 1995, RR Lyrae stars, Cambridge University Press

Smith, H.A.,0 Church, J.A., Fournier, J., Lisle, J., Gay, P., Kolenberg, K., Carney, B.W., Dick, I., Peterson, R.C., Hakes, B. 1981, PASP, 115, 43 
Struve, O., Blaauw, A. 1948, ApJ, 93, 721

Szeidl, B., Kollath, Z. 2000, ASP Conf. Ser. 203, 281

Van Hoolst, T., Dziembowski, W.D., Kawaler, S.D. 1998, MNRAS, 297, 536

Zima, W., Kolenberg, K., Briquet, M., Breger, M. 2004, Comm. in Asteroseismology (CoAst) 144, 5

\section{Discussion}

SHIBAHASHI: I suppose that, when you say something about the azimuthal order $m$, you are implicitly assuming ' $m$ ' with respect to the rotation axis. Since the magnetic model deals with the axisymmetric component with respect to the magnetic axis being oblique to the rotation axis, this nonradial component is expressed in terms of a sum of nonaxisymmetric components with respect to the rotation axis. So, in this respect, your observational claim of the presence of nonaxisymmetric components (with respect to the rotation axis) does not contradict the magnetic model.

KolenberG: Indeed, in the version of the moment method we used it is assumed that the pulsation axis coincides with the rotation axis. We used the slow-rotation approximation.

MICHEL: Is there any known correlation between the amplitude (or existence) of the blazhko effect and the projected rotational velocity $(v \sin i)$ ? If yes, this could be an argument in favor of a given type of nonradial mode possibly 'interfering' with the radial mode.

KolenberG: Projected rotational velocities of RR Lyr stars are not well known/studied (see text). Your suggestion is definitely something to explore in the future.

PRESTON: Blazhko periods projected back to the main sequence predict rotational velocities of many tens of $\mathrm{km} \mathrm{s}^{-1}$, but to my knowledge no Population II turnoff stars possess measured rotation.

PRZYBILla: Does the stellar energy distribution vary over the Blazhko cycle, i.e., does the mean energy output of the star change between pulsation cycles in the Blazhko minimum/maximum? Is there some compensation in different photometric passbands?

KolenberG: If the effective temperature range changes during the Blazhko cycle (e.g., if the RR Lyrae gets brighter at maximum because it gets hotter), then we might expect the wavelengths at which the energy is emitted to change slightly during the Blazhko cycle. I do not know whether this change has been observed and studied, and what is its size.

PRESTON: It barely changes.

WADE: You mentioned our article (Chadid et al. 2004) on the results of our magnetic field measurements of RR Lyrae, which led to the conclusion that "RR Lyrae does not show any magnetic field over the course of the 4-year cycle". I want to underscore that the Chadid et al. study, because it was extended over 4 years, explicitly ruled out the idea of a magnetic cycle of this duration (and rebutted, therefore, any argument that we observed the star at 'magnetic minumum' or somesuch). 